\title{
Probing the black hole - bulge relationship at high redshift with $\mathrm{CO}$ molecular lines
}

\author{
Xue-Bing Wu \\ Department of Astronomy, Peking University, Beijing 100871, China \\ email: wuxb@pku.edu.cn
}

\begin{abstract}
It has been suggested that the $\mathrm{CO}$ line width $(\mathrm{FWHM}(\mathrm{CO}))$ is a surrogate for the bulge velocity dispersion $(\sigma)$ of the host galaxies of high-redshift quasars, and the black hole - bulge $(M-\sigma)$ relation obtained with this assumption departs significantly from the $M-\sigma$ relation in the local universe. Based on an investigation of the correlation between the $\mathrm{CO}$ line width and the bulge velocity dispersion using a sample of 33 nearby Seyfert galaxies, we find that the formula adopted in previous studies, $\sigma=\mathrm{FWHM}(\mathrm{CO}) / 2.35$, is generally not a good approximation. By involving the galactic inclination angle $i$ as an additional parameter, we obtain a tight correlation between the inclination-corrected $\mathrm{CO}$ line width and the bulge velocity dispersion, namely, $\mathrm{FWHM}(\mathrm{CO}) / \sin \mathrm{i}=-67.16 \pm 80.18+(3.62 \pm 0.68) \sigma$. Using this new relation, we can better estimate the bulge velocity dispersion from the $\mathrm{CO}$ line width if the galactic inclination is known. We apply this new relation to nine high-redshift quasars with $\mathrm{CO}$ line detections and find that they are consistent with the local $M-\sigma$ relation if their inclination angles are around $15^{\circ}$. The possible smaller inclinations of the high-redshift quasars are preferred because of their relatively greater likelihood of detection, and are also consistent with their relatively smaller CO line widths compared to the submillimeter galaxies (SMGs) at high redshift having a similar total amount of molecular gas.
\end{abstract}

Keywords. galaxies: active — galaxies: Seyfert — quasars: emission lines — radio lines: galaxies
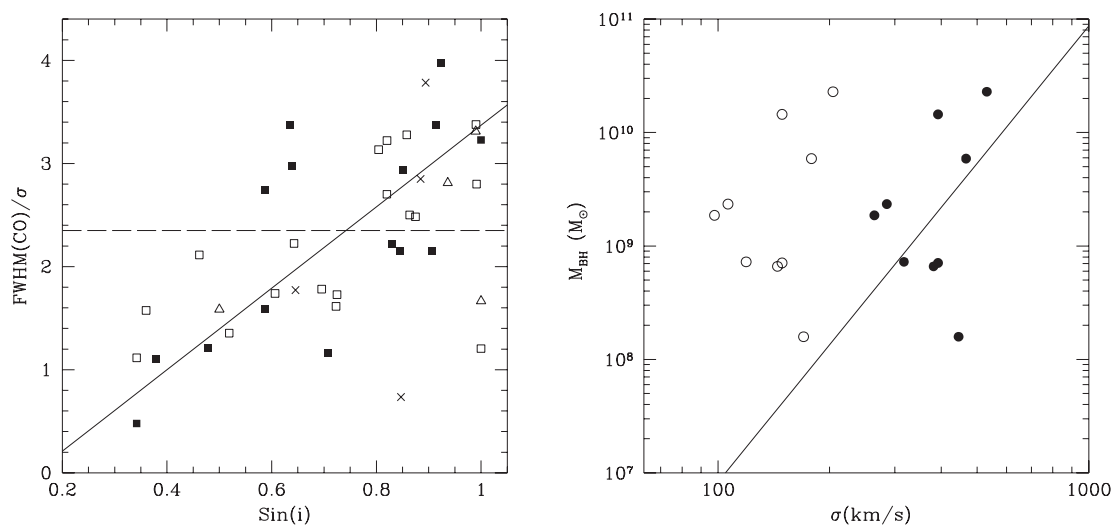

Figure 1. Left panel: The dependence of the ratio between $\mathrm{CO}$ line width and bulge velocity dispersion on the galactic inclination for Seyfert 1s (filled squares), Seyfert 2s (open squares) and radio galaxies (crosses). The solid line shows the OLS bisector fit, while the dashed line shows $\sigma=\mathrm{FWHM}(\mathrm{CO}) / 2.35$. Right panel: The $M_{\mathrm{BH}}-\sigma$ relation for high redshift quasars. The open circles correspond to $\sigma$ values derived from $\sigma=\mathrm{FWHM}(\mathrm{CO}) / 2.35$, while the filled circles correspond to $\sigma$ values derived from the inclination-corrected $\mathrm{CO}$ line widths by assuming the inclination of $15^{\circ}$. The solid line shows the local $M_{\mathrm{BH}}-\sigma$ relation.

\section{References}

Wu, X.-B. 2007, ApJ, 657, 177 